\title{
Modern Approaches to Decision-making based on Fuzzy Sets
}

\author{
Ghazi H. Shakah ${ }^{1 *}$ and V. V. Krasnoproshin ${ }^{2}$ \\ 'Ajloun National University, Jordan; g.shakah@anu.edu.jo \\ ²Belaurussain State University, Minsk, Belarus; Krasnoproshin@bsu.by
}

\begin{abstract}
Objectives: Significant progress has been made in processing the data required for enterprise management, with the development of information and computer technologies. Methods: But a deeper study of the problem of management requires the involvement of new types of information, because the economic situation is becoming more dynamic and uncertain. Mathematicians and economists should explore this tendency; develop new decision-making schemes that more fully take into account reality, while traditional formal schemes often do not correspond to factual facts. Problems that constantly arise in management due to their natural nature of uncertainty cannot always be solved with the help of traditional mathematical methods. Findings: At the same time, new theories appear, for example, the theory of fuzzy sets, which has been developing rapidly in recent times, which corresponds to these needs. Thus, it is possible to free a person from time-consuming, routine work and unleash his creative potential. Applications: All these factors make it possible to look at the prospects for the development of society in the new millennium with optimism and optimism.
\end{abstract}

Keywords: Fuzzy Decision-making, Fuzzy Sets. Methods, Modeling

\section{Introduction}

Modern society is characterized by a high level of dynamism and an abundance of information about social, economic, production systems and processes. In these conditions, management is becoming increasingly important. In order to achieve the goals set and the future prospects for the development of society, it is necessary to constantly make decisions that take into account the variability of the situation and are based on the most accessible information. The scope of management is truly comprehensive. Management developed, improved, and became more complex along with the development of human society. All changes in productive forces and production relations invariably affect the sphere of control. Therefore, it is quite natural that a revolution in the science and technology of our time leads to revolutionary changes in management.
The modern level of development of mathematics and computer science has allowed us to set and solve fundamentally new problems, which are characterized by complex relationships, large volumes of information and the need to take into account many uncertainties. Among the important objects of management can be identified enterprise. A modern enterprise is the center of human efforts aimed at quantitative and/or qualitative change of goods to meet the needs of society. In the enterprise there is a certain cyclical process ${ }^{1}$. This process is a general material scheme of economic activities, i.e. there is a production phase, a possible accumulation of goods, their transfer and a consumption phase. To manage an enterprise, it is necessary to identify a set of efforts, materialized in the behavior of the manager. Efforts are aimed at achieving a specific goal.

Currently, the economic, social and technological situation in enterprises, as a reflection of reality, is much

${ }^{*}$ Author for correspondence 
less predictable and is in a more unstable situation than a few years ago. Therefore, both at the economic level and in microeconomics, a search is under way for new approaches to the analysis of situations. These situations are characteristic of economic systems and enterprises.

Achievements in modern mathematics and artificial intelligence, along with the level of development of telecommunication and information and computer technologies, make it possible to take a fresh look at the problems of management ${ }^{2.3 .3}$ and decision making ${ }^{4}$. There was a real opportunity to automate this process from the extraction of data and knowledge to monitoring the implementation of management decisions. At the same time, it is possible to visualize and analyze the situation at all stages of decision making. To achieve these goals, it is necessary to thoroughly study and understand the global processes occurring in socio-economic systems, to analyze them in terms of achievements in formal methods and computer technologies $\frac{5,6}{6}$. But you should always remember that the direction should be from problem to methods and technologies, and not vice versa.

\section{The Problem of Modeling and Methods}

The search for effective management decisions always involves the construction of a mathematical model and the use of mathematical tools. When solving applied problems related to enterprise management, problems arise with modeling methods. This concerns the choice of formalism and the type of model. (Formalism means language to describe a model). Using a formal approach, we have the following sequence of actions:

$<$ Object task $\rightarrow$ Model $\rightarrow$ Algorithm $\rightarrow$ Programme $>$

The choice of model type is largely determined by the ability to obtain a numerical solution of the problem. To do this, there must be an algorithm that is efficient in accordance with the number of operations, the solution time, the amount of memory needed. The choice of formalism is associated with the attitude of the researcher to the information about the task. Currently, there are three fundamental possibilities: formalisms based on deterministic, probabilistic and fuzzy languages for describing reality. The main role of the program is associated with the computer implementation of the algorithm, the organization of the data structure, the presence of a user-friendly interface and the means of presenting and interpreting the result.

Let us consider some aspects related to the choice of formalisms. The meaning of the problem is determined by the following circumstances. In practice, information about the objects of the system under study is between complete confidence and chaos. This situation is called uncertainty. At any given time, the researcher is in a situation of a certain level of uncertainty. It depends on many factors. The consequence of this uncertainty is the need to use the language of "fuzzy" mathematics. In some cases, when something can be sacrificed, a deterministic formalization language or normalized fuzziness-probability is used.

These are all the possibilities that a mathematician has at his disposal when solving applied problems. Traditional formalisms have extensive experience in solving practical problems, internal techniques and a number of problems. The question arises how to account for uncertainty with the help of "fuzzy" mathematics. What are the opportunities and challenges? In what respect is it worse or better?

"Fuzzy" mathematics should proceed from the experience of traditional formalisms and make the most of it. But there must be a reverse transmission phenomenon. This means that when a transition occurs, for example, to the usual membership functions from the set $\{0,1\}$, the result should be the same as in the framework of a deterministic language (the principle of retaining feedback). With regard to the symbolism of languages, all of these formalisms differ little. But their expressiveness is very different from each other. The most expressive is the language of "fuzzy" mathematics, because it has everything that can be described in other languages, and even more. But a number of difficulties arise:

- Information volumes at least double, because the membership function appears

- It is necessary to calculate the degree of ownership of the result, when the degree of belonging of the initial information is indicated in various ways;

- Many options for the introduction of various operations on sets and numbers.

Of course, it all depends on the specific problem, but the feedback must be respected.

The level of adequacy of results largely depends on the combination of the correct choice of the type of model, 
which reflects the structural properties of the system under study, and the corresponding choice of formalism, which expresses the properties of information about the material.

Thus, modelling is a creative process. Modelling in a broad sense means replacing the system under study with another similar in some sense system, called a model and carrying out the necessary experiments with this system. The following traditional types of models can be distinguished in applied mathematics.

Analytical models are built by replacing the material carriers of the main properties of the system with abstract ones. These are mathematical symbols and various relationships between them.In this case the following questions should be answered:

- What should be used for management? It is necessary to describe the vector of controlled variables.

- How to manage? It is necessary to define a set of feasible solutions.

- What is the reason for management? The goal must be described.

- Examples of such problems are the problems of mathematical programming, graph theory, etc.

Simulation models are programs (algorithms) that implement the behaviour of the system over a long period of time. Predicted behaviour of real systems with various random actions.

Situational models: This is a prediction of the functioning of the system as a sequence of individual situations at discrete time. A set of situations is called a situational model. A set of simulation and situational models is called business games. Nowadays, computer-based decisionmaking methods are becoming increasingly recognized. These methods are based on the ideas of artificial intelligence ${ }^{7}$ and new information technologies. Among them

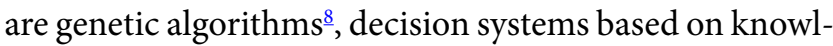
edge (expert systems) ${ }^{9,10}$, recognition systems ${ }^{11}$, Artificial Neural Networks ${ }^{\frac{12}{2}}$, and others.

Genetic Algorithms were developed based on Holland's original work ${ }^{13}$ on adaptive systems. Genetic Algorithms are stochastic optimization algorithms $s^{\underline{14}}$, which were originally motivated by the mechanisms of natural selection and genetics. What can make a genetic algorithm attractive is that its simplicity is not limited to restrictive assumptions about search space. Baseline data are the basis for generating a random set of possible solutions. This population is used to create new generation solutions. The next generation is created from the best elements of the current generation through the following genetic transformations: Selection, crossover, mutation. The expert system describes the conclusion of an expert in the subject area using the knowledge base. The knowledge base contains facts and rules relating to the subject area and the inference procedure $e^{9,15}$.

Recognition systems implement the principle of modelling in accordance with the priority. In this case, examples of objects that are carriers of an object task are indicated. If a new object appears, then in accordance with the principle of "similarity" it is assigned to one of the specified objects. Each specified object is associated with a specific solution $\frac{16}{}$.

Artificial Neural Network models the brain. This is an information processing model ${ }^{17-19}$. Note that the Artificial Neural Network implements the principle of parallel processing of information flows. Systems implemented on their basis have high throughput.

In conclusion, the use of computer methods requires a good understanding of computer and information technologies and the attraction of expert knowledge.

\section{Fuzzy Decision-making in the Management of the Enterprise}

It is generally accepted that incomplete or partial reliability is a typical situation in mathematical applications. This is due to the fact that most of the problems are separate fragments of reality. When describing a fragment, it is necessary to diverge from certain information that will be present in the description of the objects being formalized. This happens when the quantitative measurement of certain characteristics is impossible or they are subjective and so on. In such situations, the methodological aspects of mathematics take on paramount importance. The main question concerns the choice of mathematics from the set of its possible variants (deterministic, probabilistic, fuzzy, etc.) for the formalization and solution of the problem ${ }^{20}$. It should be understood that the wrong choice is the cause of the inability to prove the results, their processing and leads to an overestimated significance.

In such conditions, fuzzy mathematics is widely used in those areas where the formalisms of ordinary mathematics are not applicable due to the lack of an appropriate 
language. This situation is typical for most decision making and management tasks. Let us consider the problem of decision making, which can be formulated as follows.

Let the set of possible solutions $\mathrm{K}$ be numbered by the numbers $\mathrm{k} 1, \ldots, \mathrm{kl}\left(\mathrm{k}_{\mathrm{i}} \in \mathrm{N}\right)$, ordered (assume that $\mathrm{k}_{1} \leq \mathrm{k} 2$ $\leq \ldots \leq \mathrm{k}_{\mathrm{l}}$ ) and the range of decision $\mathrm{X}$ will be an arbitrary non-empty set of objects. Suppose that for each solution with the number $\mathrm{k}_{\mathrm{i}} \in \mathrm{K}$, a certain subset $\mathrm{Xi} \subseteq \mathrm{X}$ ( $\mathrm{i}=$ 1..l) can be specified in an objective correspondence. It is required to find an algorithm that is defined on the whole set $\mathrm{X}$ and the result of the algorithm can be interpreted in terms of possible solutions from $\mathrm{K}$.

This statement is an obvious basis for management tasks. Having prepared for the solution of the problem, it is necessary to associate on the set $\mathrm{X}$ every possible solution from $\mathrm{k}_{\mathrm{i}} \in \mathrm{K}$ with some control action. In this case, the construction of algorithm A is somewhat more complicated.

It should be noted that there are other provisions on the decision-making tasks. They mainly differ in the choice of the space of possible solutions $\mathrm{K}$ and as a consequence, in the assumptions about the correlation between $\mathrm{X}$ and $\mathrm{K}$. In most of these statements, the question is to construct a function. In the case of ambiguity and due to the large difference in the options for implementing relational operations on the set $\mathrm{X}$, this function can be constructed in different ways.

All existing variants of the mentioned task differ in the volumes and methods of specifying information on the set $\mathrm{X}$. The subsets $\mathrm{X} 1, \ldots, \mathrm{Xl}$ can be specified in full or in partially through the finite subsets $\mathrm{X}_{\mathrm{i}}^{0} \subset \mathrm{Xi}$. In both cases, for each object $\mathrm{x}$, the corresponding solution $\mathrm{K}_{\mathrm{i}}(\mathrm{i}$ $=1 . . \mathrm{l})$ is known. Obviously, the problem is degenerate if full information is known, because it is solved by a simple recall.

There are two fundamentally different ways of specifying information - through the rules and use cases (examples). These methods are methodologically related. The second precedes the first and is used when we have only a description of $\mathrm{x}$ with a known suitable solution $\mathrm{K}_{\mathrm{i}}$. Any other information is missing. The first method implements a logical description of the subsets $\mathrm{X}_{\mathrm{i}}\left(\mathrm{X}_{\mathrm{i}}^{0}\right)$ using the set of rules (predicates) P1, ..., Pl. They are as follows:

$$
P_{i}(x)=\left\{\begin{array}{l}
1, \text { if } x \in X_{i}\left(X_{i}^{0}\right), \\
0, \text { otherwise }
\end{array}\right.
$$

Change from logic way to precedent is not so difficult. Using $\mathrm{P} 1, \ldots, \mathrm{Pl}$ it is necessary to build subsets:

$$
\mathrm{X}_{\mathrm{i}}=\left\{\mathrm{x} \in \mathrm{X} \mid \mathrm{P}_{\mathrm{i}}(\mathrm{x})=1\right\}
$$

Such a change is generally technical in nature. Its meaning lies in the equivalent transformation of the original rule set $\mathrm{Pi}$ into some form. This form is called the Disjunctive Normal Form (DNF). Each conjunction of such a formula is an object $\mathrm{x} \in \mathrm{X}_{\mathrm{i}}\left(\mathrm{X}_{\mathrm{i}}^{0}\right)$. Consider the decision-making problem in the above statement with the additional assumption that information about subsets is given in part - using finite $\mathrm{X}_{\mathrm{i}}^{0}$ subsets and through precedents. Consider algorithm A, which solves the problem. In general, A realizes reflection.

$\mathrm{A}: \mathrm{X} \rightarrow \mathrm{K}$

This reflection in the final passage $U \mathrm{X}_{\mathrm{i}}^{0}$ must satisfy the following constraint:

$\forall \mathrm{x} \in \mathrm{X}_{\mathrm{i}}^{0}\left(\mathrm{~A}(\mathrm{x})=\mathrm{k}_{\mathrm{i}}\right.$

Because following the assumption:

$$
\bigcup_{i=1}^{\prime} X_{i}^{0} \subset \bigcup_{i=1}^{\prime} X_{i} \subseteq X
$$

There is no reason to assume that:

$\mathrm{X}_{\mathrm{i}} \cap \mathrm{X}_{\mathrm{j}}=\varnothing$ if $\mathrm{i} \neq \mathrm{j}$.

It is obvious that in this case there exists an object $\mathrm{x} \in \mathrm{X}$, for which $\mathrm{A}(\mathrm{x})$ is not defined or has more than one number from $\mathrm{K}$ as a solution. To avoid uncertainty, the solution space of algorithm A can be extended with additional characteristics.

$$
M=\left(\mu_{A}\left(k_{1}\right), \ldots, \mu_{A}\left(k_{1}\right)\right) \text {. If in this case } \mu_{A}\left(k_{i}\right) \in[0,1],
$$

Then the vector $\mathrm{M}$ can be considered as the vector of belonging of the corresponding $\mathrm{x} \in \mathrm{X}$ to the subsets $\mathrm{Xi}$ ( $\mathrm{i}=$ $1 \ldots, 1)$. Then, as soon as $\mathrm{K}$ is ordered, we must correct its vector representation $(\mathrm{K} 1, \ldots, \mathrm{Kl})$ and construct an algorithm as:

$\mathrm{A}: \mathrm{X} \rightarrow \mathrm{K} \times \mathrm{M}$

If the following condition is met: $\forall \mathrm{x} \in \mathrm{X}_{\mathrm{i}}^{0}\left(\mathrm{~A}(\mathrm{x})=\left(\left(\mathrm{k}_{\mathrm{l}}, \mu_{\mathrm{A}}\left(\mathrm{k}_{\mathrm{l}}\right)\right), \ldots,\left(\mathrm{k}_{\mathrm{l}}, \mu_{\mathrm{A}}\left(\mathrm{k}_{\mathrm{l}}\right)\right)\right.\right.$ and $\mu_{\mathrm{A}}\left(\mathrm{k}_{\mathrm{i}}\right)=1$

To calculate the vector of membership $M$. you can use the method of generalization. In our notation, it will look like this: 


$$
\mu_{A}\left(k_{i}\right)=\max _{x^{\prime} \in X_{i}^{0}} \mu_{X_{i}}\left(x, x^{\prime}\right\}
$$

Where $\mu_{\mathrm{xi}}:\left(\mathrm{x}, \mathrm{x}^{\prime}\right) \rightarrow[0,1]$, is defined on the whole set $\mathrm{X}$ and can be calculated for each $\mathrm{x}^{\prime} \in \mathrm{X}_{\mathrm{i}}^{0}$. The latter reflection is naturally called the function of belonging to the subsets Xi.

In fact, with such a transformation of algorithm A, we replace the task of calculating numbers from $\mathrm{K}$ with a wider and more natural task of calculating the membership function for each number $\mathrm{K}_{\mathrm{i}}$ from a fixed sequence $\mathrm{K}$. Obviously, in this case, the result of algorithm A can be considered a fuzzy number, and the algorithm can be considered as a fuzzy algorithm ${ }^{21,22}$. In the transition to fuzzy algorithms, some natural basis for comparing them appears. This follows from the following definition.

Algorithm $A_{1}$ dominates $A_{2}\left(A_{1} \geq A_{2}\right)$, if

$\forall \mathrm{x} \in \mathrm{X}, \forall \mathrm{k}_{\mathrm{i}} \in \mathrm{K}\left(\mu_{\mathrm{A} 1}\left(\mathrm{k}_{\mathrm{i}}\right) \geq \mu_{\mathrm{A} 2}\left(\mathrm{k}_{\mathrm{i}}\right)\right)$

It should be noted that for the problem under consideration there are so-called canonical (universal) algorithms. If the information is specified in the rules, then this is the permission method. When precedents are used for this purpose, then this is a processing algorithm. If the canonical algorithm is designated as $\mathrm{A} 0$, then in our notation the result of its work can be determined by the condition:

$$
\mu_{A_{0}}\left(k_{i}\right)=\left\{\begin{array}{l}
1, f x \in X_{i}^{0}\left(P_{i}(x)=1\right) \\
0, \text { otherwise. }
\end{array}\right.
$$

However, there are at least two drawbacks that limit the applicability of A0. The function $\mu \mathrm{A} 0:\left(\mathrm{x}, \mathrm{x}^{\prime}\right) \rightarrow[0,1]$ and the algorithm $\mathrm{A} 0$ itself are not fuzzy. In addition, $\mu \mathrm{A} 0$ may be undefined for predicate computing languages with 1 or higher order. Nevertheless, A0 can be used for comparison with the developed algorithm A. At least, there is no doubt that the use of A0 for solving the problem is legitimate and sufficient. Therefore, any proposed algorithm A should dominate A0 and only in this case it has the right to exist. Of course, the algorithm may have other useful properties.

A general scheme for constructing such algorithms A was proposed in ${ }^{23,24}$ and it was shown that: Each algorithm A solves the problem posed and dominates the canonical algorithm $\mathrm{A} 0 \underline{25}$.
Thus, there is the possibility of justifying fuzzy algorithms arising from the transition from deterministic to fuzzy processing of results.

So, at present there is the necessary set of methodological, mathematical and computer knowledge to successfully put into practice the formal methods of enterprise management.

\section{Conclusion}

The world community is experiencing an era of rapid development. There is a huge concentration of capital, the development of high-tech production, a massive transfer of people. In a short time, individual countries are moving from underdeveloped to highly developed. The world is saturated with huge amounts of information that is constantly increasing. To survive, people, companies, countries constantly make decisions based on various factors.

Decision making requires automation, but this cannot be done without a transition to the formal level, that is, formal methods are being introduced into weakly formalized, from the point of view of mathematics, spheres. The existing standard mathematical schemes are not suitable, new approaches and theories appear. We are moving from an understanding of the world, when decision-based statistics have been generated, to a world of uncertainty, when processes change rapidly and phenomena are not measured quantitatively. At the same time, we are witnessing the achievements of scientists in the development of new languages to describe reality, that is, fuzzy mathematics and success in artificial intelligence.

Advances in information and computer technology deserve special attention. In fact, the problem of creating a functional space for information was solved. Like airlines, the whole world is covered by an information and computer network. Existing technologies based on data and knowledge bases allow you to store and quickly process huge amounts of information. There is success in creating natural language interfaces. All these factors create good prerequisites for the realization of the creative potential of scientists who are able to solve their tasks.

\section{Funding}

This research is funded by the Deanship of Research and Graduate Studies in Ajloun National University Ajloun, 26810, Jordan. 


\section{References}

1. Kaufmann A, Gil Aluja J. Introduction de la Teoria de los Subconjuntos Borrosos a la Gestion de las Empresas. Santiago de Compostela: Milladoiro; 1986.

2. Zadeh LA, Bellman RE. Decision-making in a Fuzzy Environment. Managem. Sci. 1970; 17:141-64. https://doi. org/10.1287/mnsc.17.4.B141

3. Edith Cowan University Research Online ECU Publications Pre. Approaches to decision making. Richard J. McKenna Edith Cowan University; 2011.

4. Orlovsky SA. Decision-making with a Fuzzy Preference Relation. Fuzzy Sets and Systems. 1978; 1(3):155-67. https://doi.org/10.1016/0165-0114(78)90001-5

5. Gil Aluja J. Towards a new concept of economic research. J. Fuzzy Economic Review; 1995. p. 5-23. https://doi. org/10.25102/fer.1995.01.01

6. Popper K. The logic of scientific discovery. (Rout ledge Classics). 2017; 56.

7. Thomason RH. Logic and artificial intelligence. 2002. http:// web.eecs.umich.edu/ rthomaso/documents/lai/lai.pdf

8. Shakah G, Al-Oqaily A. Solving non-linear optimization problems using parallel genetic algorithm. 8th International Conference on Computer Science and Information Technology (CSIT); 2018. https://doi.org/10.1109/ CSIT.2018.8486176

9. Kumar A. Artificial intelligence: A 'Real-World' problem solver in the midst of paradoxes. 2018.

10. Sedlak O. Developing expert systems in decision making by applying the fuzzy set theory. Advances in Environmental Science and Energy Planning. 2015. p. 41-50.

11. Shakah G. A new method for solving hard diagnosis problems. Computer Engineering and Intelligent Systems. 2019; 10(1):13-9.

12. Krenker A. Introduction to the Artificial Neural Networks. Artificial Neural Networks - Methodological Advances and Biomedical Applications. 2011. p. 3-17. https://doi. org/10.5772/15751

13. Shakah G. The problem of image segmentation and denoising methods and various approaches to its solution.
International Journal of Engineering and Technology. 2018; 7(4):5297-301.

14. Golberg DE. Genetic Algorithms in search, optimization and machine learning. Reading, Mass: Addison-Wesley; 1989.

15. Shakah G, Al-Sharo Y, etc. Classification of big data: Machine learning problems and challenges in network intrusion prediction. International Journal of Engineering and Technology. 2018; 7(4):3865-9.

16. Hu B. Analysis, design and evaluation of man-machine systems. Elsevier. 2015.

17. Shakah G, et al. Decision making system for operative tasks. International Conference on Pattern Recognition and Information Processing; 2009. p. 272-5.

18. McCord Nelson M, Illingworth WT. A practical to guide to neural nets. Reading, MA: Addison Wesley; 1991.

19. Masters T. Practical Neural Network Recipes in C++. Academic Press: New York; 2010.

20. Shakah G, Krasnoproshin VV, Obraztsov VA. Fuzzy algorithm in Decision-making problem. Proc of 2nd Congress of SIGEF, v.2; Santiago-de-Compostela. p. 283-96.

21. Hu J, Zhang Y. Multi-criteria decision making method based on possibility degree of interval type-2 fuzzy number. Knowledge-based Systems. 2013; 43:21-9. https://doi.org/10.1016/j.knosys.2012.11.007

22. Wong CK. Fuzzy points and local properties of fuzzy topology. J Math Anal Appl. 1974 May; 46(2):316-28. https://doi. org/10.1016/0022-247X(74)90242-X

23. Krasnoproshin VV, Shakah G, Monitoring and management of natural territorial complexes. Advances in Computing. 2012; 2(1):1-5. https://doi.org/10.5923/j.ac.20120201.01

24. Shakah G, Vissial H. Decision support system based on subject collections: Architecture and application. Journal of Computational Optimization in Economics and Finance. 4(2/3):177.

25. Runkler T, Coupland S. Interval type-2 fuzzy decision making. International Journal of Approximate Reasoning. 2017; 80:217-24. https://doi.org/10.1016/j.ijar.2016.09.007 